CLAWAR 2018: 21st International Conference on Climbing and Walking Robots and the Support Technologies for Mobile Machines, Panama City, Panama, 10-12 September 2018

\title{
TOWARDS SAFE INSPECTION OF LONG WELD LINES ON SHIP HULLS USING AN AUTONOMOUS ROBOT *
}

\author{
GABRIELA GALLEGOS GARRIDO, TARIQ SATTAR, MICHAEL CORSAR ${ }^{\dagger}$ \\ London South Bank University, London South Bank Innovation Centre, Granta Park \\ Great Abington, Cambridge CB21 6AL, UK. \\ RICHARD JAMES \\ Innotec $U K, R \& D$ department, Hildersham Road \\ Cambridge, Cambridgeshire CB21 6DR, UK. \\ DJELLOUL SEGHIER \\ NDT Consultants LTD, R\&D department, Siskin drive \\ MiddleMarch House, Coventry CV3 4FJ, UK.
}

\begin{abstract}
Inspection of ship-hull welds must be carried out in accordance with classification society guidelines during manufacture and when in service. Failure of ship-hull welds can result in the loss of a vessel, loss of life and pollution of the environment. Typically weld lines are inspected using ultrasound NDT techniques which are labour intensive, expensive and often hazardous to operators. To remain competitive shipbuilders and inspection companies need to reduce costs while maintaining or improving inspection quality. Automated inspection can achieve this, but current systems are complex, expensive and not suited to shipyard operation. AWI is a novel robotic autonomous system (RAS) that uses magnetic adhesion to climb ship hulls and autonomously track weld lines while performing ultrasonic scans of the welds. Communication of the inspection data is transmitted wirelessly to the inspector sited remotely. This addresses a key challenge in overcoming the payload limitations of current climbing robots. Therefore, AWI is equipped with rugged, fast and secure wireless communications, which robustly integrates with the robot hardware for industrial service ${ }^{\S}$.
\end{abstract}

\section{Introduction}

\subsection{Objectives of the research}

All ship hull welds must be inspected prior to a vessel entering service and at periodic intervals during its service life as per guidelines issued by the

\footnotetext{
${ }^{*}$ This work is supported by Innovate UK Project No: TP102906

${ }^{\dagger}$ Work partially supported by ESPRC grant EP/R005451/1

$\S$ The project website: www.awi-weldinspectionrobot.co.uk
} 
classification societies [8]. Ultrasound based methods are widely accepted as standard means for non-destructive inspection of welds. For each ship hull many 100s of metres of weld may require inspection. Due to the nature of the hull and the ship yard environment inspections can be hazardous, often requiring rope access and scaffolding to reach the weld lines. Safety and productivity can be greatly enhanced throw automation of the hull inspection. Therefore, the present work outlines the development of a new climbing robot designed to carry out phased array ultrasound inspection on ship hulls.

\subsection{Outline of robot requirements}

AWI is a climbing robot, using magnetic adhesion, designed specifically to inspect weld lines. Its primary operating environment is the external hull of newly manufactured ships, focussing particularly on areas which are difficult to access by humans. The aim is to remove the requirement for inspection by rope access and scaffolding which will improve safety for the inspector and greatly reduce the cost of inspection.

In order to move the state of the art forward and to be attractive to industry the robot and inspection system must be able to autonomously follow weld lines while carrying out ultrasound inspection. When defects are identified they must be marked so that intervention can be carried out. The robot must travel at high speed between inspection areas but also be able to travel at a lower rate determined by the inspection system.

\section{Industrial climbing robots}

The design of a climbing robot for industrial applications depends on several factors influenced by its operating environment and the task it must carry out. Research activities on wall climbing robot has been carried out for several years now [1][3][4], but inspection of weld lines on large ship hulls remains a challenge. Payload capacity, mobility, adhesion safety and energy consumption are the primary factors influencing design choices [5]. For climbing on non-ferrous surfaces, systems tend to employ either energy intensive suction or dynamic vortex adhesion methods. For ferrous structures, the choice is permanent magnets or electromagnetic adhesion. Electromagnets offer the ability to control the magnetic on command but require constant energy consumption to maintain their magnetic field [6]. To overcome payload limitations and power consumption problems, it was necessary to use permanent magnets mounted in the robot wheels. This will also prevent the robot from falling in the case of a power failure. 
The robot must be capable of climbing on both steeply sloped and vertical surfaces and be able to transition across different planes. Therefore, a force analysis has been carried out to ensure stability of the robot across all climbing modes to avoid sliding and roll-over failure. Figure 1 shows a free body diagram of the robot operating on a vertical plane, where the robot weight is $\mathbf{G}$, the pitch between the centre of the front and rear wheels is $\mathbf{L}$, and the centre of gravity from the climbing surface is $\mathbf{h}$. The robot acceleration is $\mathbf{a}$, and the coefficient of surface friction $\boldsymbol{\mu}$ (wheels-surface). To avoid slipping, the total adhesion force $\mathbf{F}_{\mathbf{t}}$, must satisfy Equation (1):

$$
F_{t} \geq \frac{(G+m a)}{\mu}
$$

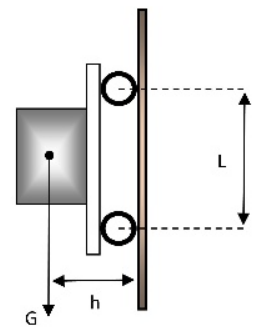

Figure 1 . Free body diagram of a robot climbing a vertical plane.

According to [2], by taking the moment about point $\mathrm{G}$ from Figure 1, the required force can be found. Therefore,

$$
F_{t} \geq \frac{G x h}{L}
$$

To prevent roll-over, the centre of gravity of the robot should be designed to be as close to the wall as possible and the wheel base should be as long as possible.

\section{Magnetic adhesion system}

The adhesion force for AWI is generated by four magnetic wheels. In order to ensure that the target adhesion force is achieved numerical simulations were carried out using COMSOL Multiphysics software. Each wheel is composed of four segments of N35 neodymium magnet of $20 \mathrm{~mm}$ thickness. The outer diameter of the wheel is $100 \mathrm{~mm}$ and the inner diameter is $35.70 \mathrm{~mm}$.

The magnets are surrounded by a $10 \mathrm{~mm}$ thickness stainless steel on both sides to keep the magnets aligned, plus $4 \mathrm{~mm}$ of rubber for protection. The ship hull has been simulated as $20 \mathrm{~mm}$ steel plate. The key properties of the materials used in the simulation are listed in Table 1 . The adhesion force achieved per wheel is $82.485 \mathrm{~N}$, which corresponds to $8.4 \mathrm{Kg}$ of pull. The distance $(\mathrm{L})$ between wheels 
is $150 \mathrm{~mm}$ and horizontally the distance is $430 \mathrm{~mm}$. Hence, the total adhesion force is computed as $330.70 \mathrm{~N}$, i.e. $33.72 \mathrm{Kg}$ pull.

\begin{tabular}{lcc}
\multicolumn{1}{c}{ Table 1. Values used for FEA simulations with N35 and N42 magnets. } \\
\hline \multicolumn{1}{c}{ Properties } & N35 & N42 \\
\hline Magnetic induction intensity of magnets $\mathbf{B}_{\mathbf{r}}(\mathbf{T})$ & 1.13 & 1.31 \\
Magnetic Coercive force $\mathbf{H}_{\mathbf{c b}}(\mathbf{K A} / \mathbf{m})$ & 868 & 915 \\
Intrinsic Coercive force Hci $(\mathbf{K A} / \mathbf{m})$ & 955 & 955 \\
Magnetic energy product HB $\left(\mathbf{K J} / \mathbf{m}^{3}\right)$ & 263 & 318 \\
Relative permeability of magnets $\left(\boldsymbol{\mu}_{\mathbf{r}}\right)$ & 1.05 & 1.05 \\
Relative permeability of wheels steel enclosure & 1 & 1 \\
Relative permeability of steel climbing surface & 1500 & 1500 \\
\hline
\end{tabular}

In further simulations the grade of the magnets was changed to higher intensity N42 magnets. This achieves an adhesion force of $110.50 \mathrm{~N}$ (11.26Kg) per wheel. Therefore, with four wheels the total adhesion force of AWI is $444.44 \mathrm{~N}$ (45.32Kg).
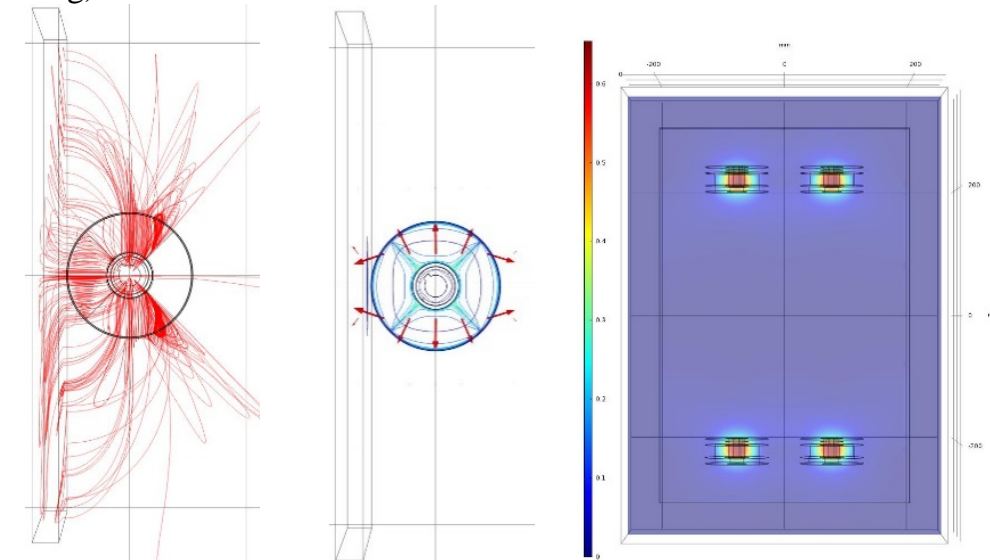

Figure 2. Results on COMSOL: a) Magnetic flux streamlines. b) Direction of the magnetic flux of the four magnets (polarisation of magnets). c) Magnetic flux density norm (four wheels).

\section{Design of the climbing robot}

\subsection{Mechanical hardware}

The main chassis of AWI is constructed from folded and welded 316 stainless steel with a sealed marine grade anodised aluminium lid. The drive shafts are all fitted with double acting lip seals made of Therban HNBR, which when combined with IP67+ electrical connectors ensure that AWI remains watertight.

- Dimensions of AWI are L: 410mm x W: 472mm x D: $210 \mathrm{~mm}$

- Weight 25kg - Wheelbase of $150 \mathrm{~mm}$ - Track of $430 \mathrm{~mm}$

- $\quad$ Powered by 40V DC from control box 


\subsection{Electronics hardware}

Autonomous robots commonly use embedded software to operate, but AWI was designed to function using a custom made desktop application written in C\#. The main advantage of this approach is that loosely-bound code allows for future features or modifications to be made without impacting other areas of code. This is possible via a device server, which is connected to a USB hub and Ethernet switch. Some of the components on AWI work on RS485 and RS232 protocols, which are converted to USB to allow a desktop computer to identify the components as COM ports. The Ethernet switch is connected to an industrial wireless card, which has a 3 channel rugged antenna operating on $2.4-5 \mathrm{GHz}$ frequency. Figure 3 illustrates the overall hardware connections within the AWI robot.

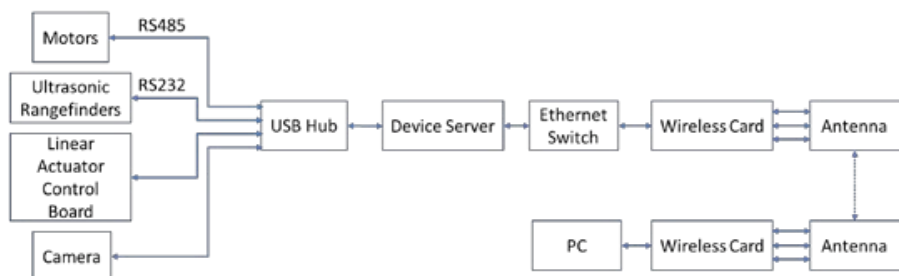

Figure 3. Diagram of hardware connections within the AWI robot.

The optical sensing of the weld lines is achieved using a green 2D laser line generator and an HD USB camera mounted in adjustable holders. The information required by the weld following algorithm is a frame captured by the camera, which applies a colour filter to the image given user selectable RGB values and then pixel detection to highlight weld area.

The NDT inspection equipment is mounted at the front of the robot. This includes an ultrasound phased array probe and a Hengstler optical encoder (more details of the inspection system is provided in section 5). Position data from the encoder is fed directly to the NDT system rather than using the motor driven encoders in order to minimise the problem of the driven wheels slipping.

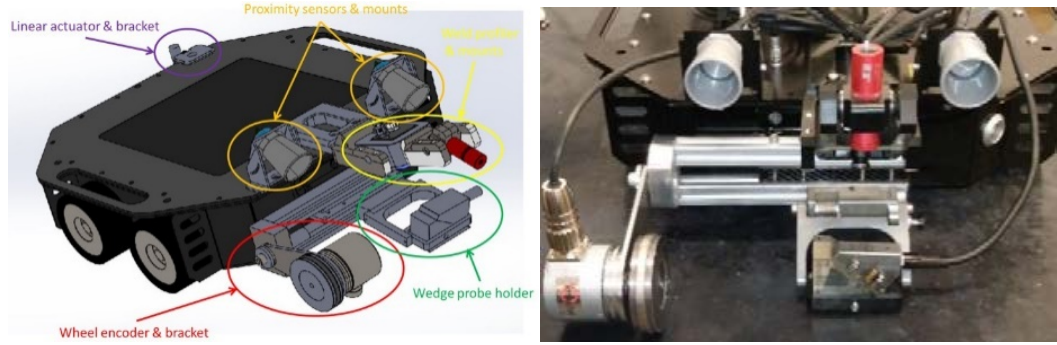

Figure 4. AWI's NDT payload. 


\subsection{Robot software and control}

The purpose of AWI is to automatically follow long welds while carrying out inspection, however, the user interface also allows operators to manually drive the robot. Image frames from the front mounted camera are displayed in bitmap format.

Weld line detection is achieved by processing each camera frame using the AForge.Net framework. The image is first filtered to remove RGB colour outside the desired threshold. The resulting monochromatic image is shown in Figure 6. The raw bitmap data is then scanned, and the peak $\boldsymbol{y}$ pixel is selected to identify the centre point of the weld.

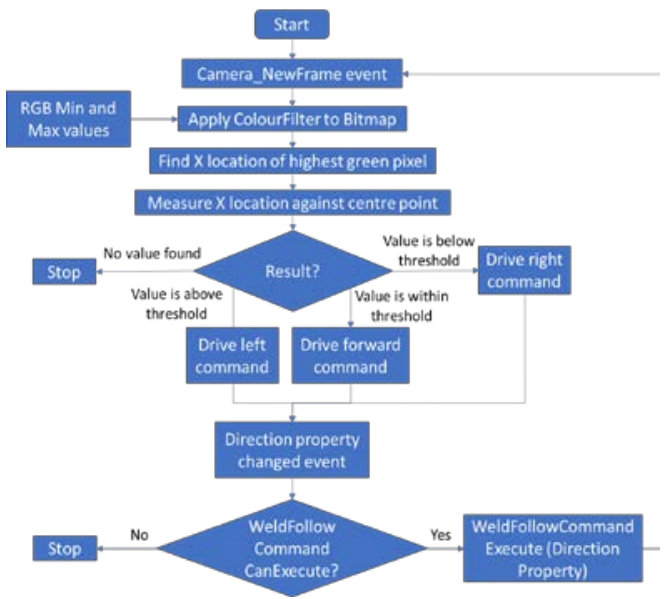

Figure 5. Weld following algorithm flow chart.

The weld centre is then compared to the centre point of the camera resolution. The difference between the two is then used as the feedback error to correct the robot direction. The weld following algorithm is described in the flowchart in Figure 5.

\section{Experimental results}

\subsection{Performance of the climbing robot}

After assembly was completed (see fig. 4) a full system test of the robot performance was carried out on a horizontal and vertical test wall (see fig. 6a) at InnoTecUK. The wall features around 36 different defects embedded in the weld, such as cracks, lack of penetration, and discontinuities. The wireless communication was robustly established and data could successfully be retrieved including visual information of the weld and the laser profiler (see fig. 6b). During 
inspection AWI's speed was set at less than $50 \mathrm{~mm} / \mathrm{sec}$, which is suitable for weld inspection. AWI's maximum speed achieved when not inspecting the weld and by teleoperation using a remote control was around $150 \mathrm{~mm} / \mathrm{sec}$.

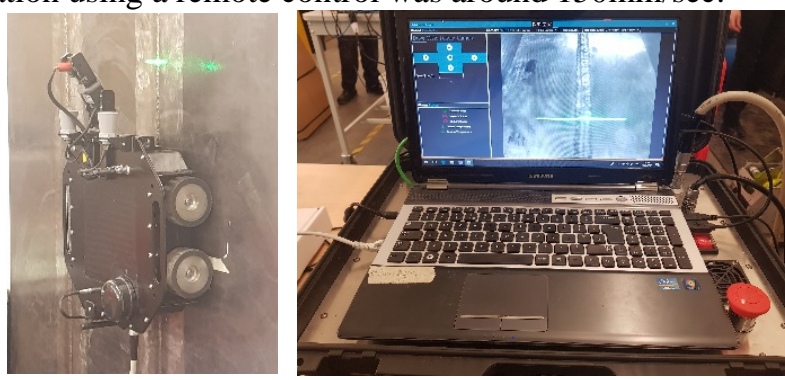

Figure 6. AWI’s Trials.

AWI was able to localise itself along the weld based on the feedback provided from the wheel encoder. The emergency stop button feature allows the operator to cut the power of the whole system and stop the robot if safety related problems are encountered. Despite being powered down, the robot will maintain its position due to its magnetic wheels, which do not require any power to work. Furthermore, AWI was shown to be able to physically mark the position of the defects as they were encountered.

\subsection{Defect detection using the on-board inspection system}

The inspection system used during the trials was a conventional phased array ultrasound system. Calculations were made to determine the wedge angle and the full and half skip distances required for full inspection of the weld line, taking into account the weld thickness (fig. 7a).
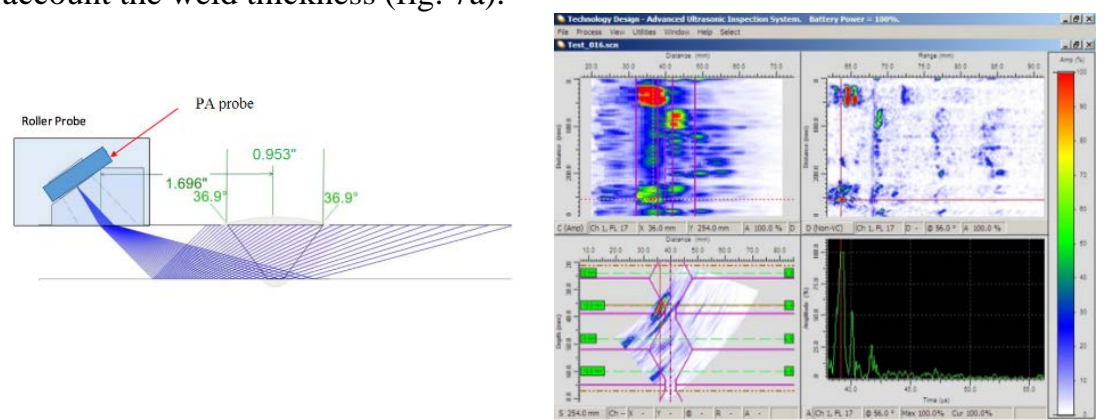

Figure 7. a) PA probe is mounted in the roller probe at $19^{\circ}$. b) Example of c-scan measured from the weld showing identification of defects.

With such an angled wedge and distance from the weld line inspection of the weld was possible and all the defects were correctly identified (fig. 7b). 


\section{Conclusions and future work}

We presented a successful integration of a phased array ultrasound inspection system on a climbing robot and demonstrated that our method could effectively detect defects in welds. Communication between the robot and the remote computer is completely wireless. As future work, we intend to reduce the chassis weight to mount a high capacity battery for at least five hours of autonomy. Our initial demonstration uses also an umbilical to provide the couplant for the inspection system. Removing the umbilical using a dry couplant as in [7] will allow AWI to operate fully wireless, so further work will be carried out to find a suitable substitute for the couplant. Finally, we will trial AWI under realistic conditions on real ship hull at several meters from the remote computer, in a range of light conditions and poor weather.

\section{Acknowledgments}

This is work was sponsored by an InnovateUK (No: TP102906) fund and ESPRC grant (EP/R005451/1), with the following partnership (in UK): London South Bank University (LSBU/LSBIC), Innovative Technology \& Science Ltd (InnoTecUK) and NDT Consultants Ltd.

\section{References}

1. Howlader, O.F. \& Sattar, Tariq. (2015). "Development of magnetic adhesion based climbing robot for non-destructive testing”, CEEC2015.

2. Howlader, O.F. \& Sattar, Tariq. (2015). "Novel Adhesion Mechanism and Design Parameters for Concrete Wall-Climbing Robot”, IntelliSys2015.

3. S. Nansai, R. Elara Mohan (2016). "A Survey of Wall climbing robots: Recent Advances and challenges”, Robotics2016, 5,14.

4. M. Armada, P. Santos, M. Jimenez \& M. Prieto (2003). "Application of Clawar Machines”, International Journal of Robotics Research, vol. 22, no. 3, pp. 251-259, 2003.

5. F. Manuel \& J. Tenreiro (2010). "A Survey of Technologies and Applications for Climbing Robots Locomotion and Adhesion”, INTECH.

6. H. Prahlad, R. Pelrine, S. Stanford, J. Marlo \& R. Kornbluh (2008). "Electroadhesive robots wall climbing robots enabled by a novel, robust, and electrically controllable adhesion technology". IEEE ICRA: 3028-3033.

7. S.C. Mondal, T.P.Sattar \& T. Pattel (2010). "Remote mobile vehicle and its suitable non-destructive testing (NDT) inspection methods for the melt Weld inspection", CLAWAR2010.

8. 'Non-destructive testing of ship hull steel welds', International Association of Classification Societies, IACS Rec. 1988/Rev. 12007 